\title{
MICROBIOLOGICAL AND MICROSCOPIC ANALYSIS OF THE PULP OF NON-VITAL TRAUMATIZED TEETH WITH INTACT CROWNS
}

Kely Firmino BRUNOํㅗ Ana Helena Gonçalves de ALENCAR ${ }^{2}$, Carlos ESTRELA ${ }^{3}$, Aline de Carvalho BATISTA ${ }^{4}$, Fabiana Cristina PIMENTA ${ }^{5}$

1- MSc, Assistant Professor, Department of Endodontics, Paulista University, Goiânia, GO, Brazil .

2- PhD, Associate Professor, Department of Endodontics, Federal University of Goiânia, GO, Brazil.

3- PhD, Full Professor, Department of Endodontics, Federal University of Goiás, Goiânia, GO, Brazil .

4- PhD, Associate Professor, Department of Oral Pathology, Federal University of Goiás, Goiania, GO, Brazil.

5- PhD, Full Professor, Department of Microbiology, Federal University of Goiás, Goiânia, GO, Brazil.

Corresponding address: Kely Firmino Bruno - Rua 19 nº 322 - Ed. Mirador, apto 804 - Setor Central - 74030-090 - Goiânia, GO - Brazil - e-mail: drkelybruno@bol.com.br

Received: July 2, 2008 - Modification: August 31, 2008 - Accepted: November 9, 2008

\begin{abstract}
$O$

bjective: This study evaluated the presence of microorganisms and analyzed microscopically the pulp of 20 traumatized human teeth with intact crowns and clinical diagnosis of pulp necrosis, based on the association of at least three of the clinical criteria: crown discoloration, negative response to thermal and electric pulp vitality tests, positive response to vertical and horizontal percussion, pain on palpation or mobility. Material and Methods: Microbiological collection was performed from the root canals to evaluate the presence of microorganisms. The pulp samples were stained with hematoxylin and eosin (H.E.) for histological evaluation of possible morphological alterations. Results: Analysis of results was performed by statistical tests (linear regression test and diagnostic analysis) and subjective analysis of the sections stained with H.E. and revealed that only $15 \%$ of the sample did not exhibit microbial development. The time elapsed between dental trauma and onset of endodontic intervention ranged from 15 days to 31 months; the percussion test presented high sensitivity $(80 \%)$ for detection of microorganisms in the root canal of traumatized teeth; 3 teeth (15\%) did not present pulp tissue, being characterized as complete autolysis; analysis of pulp samples was performed on the other 17 cases, among which 3 (15\%) exhibited partial necrosis without possibility of repair and 14 presented complete necrosis; none of the clinical criteria employed for the diagnosis of pulp necrosis in traumatized teeth was pathognomonic. Conclusions: The present results allowed the following conclusions: with regard to microbiological findings, $85 \%$ of teeth presented microorganisms in the root canal, despite the presence of an intact crown. Concerning the microscopic findings, $100 \%$ of traumatized teeth presented pulp necrosis; the pulp vitality tests based on pulp response to heat, cold and vertical percussion were the most reliable to diagnose pulp necrosis in traumatized teeth.
\end{abstract}

Key words: Trauma. Pulp necrosis. Microbiology. Microscopy.

\section{INTRODUCTION}

The accurate diagnosis and proper treatment of dental trauma is a challenging area in dentistry. Traumatic injuries to the pulp and periodontal structures produce damages that may impair the esthetics and function of the stomatognathic system, in addition to causing serious emotional and psychological problems to the patient ${ }^{7,14}$.

The main pulp changes secondary to dental trauma are pulp necrosis, root canal obliteration and resorption ${ }^{5,8}$. Since pulp necrosis is the most frequent ${ }^{8,16}$, early diagnosis is necessary before microbial invasion and the onset of external resorptions, for achievement of a favorable prognosis. However, this diagnosis is limited and may present failures, since the diagnostic methods available to determine the pulp status after trauma are not precise or standardized ${ }^{5,13}$.

Some criteria have been suggested to diagnose pulp necrosis in traumatized teeth, such as the negative response to pulp vitality tests, progressive discoloration of the crown, periapical radiolucency, interruption of root formation or positive response to the vertical percussion test ${ }^{6}$. However, the criteria are not standardized and may have different meanings along the period after trauma ${ }^{19}$, giving rise to insecurity as to the adequate moment for endodontic intervention.

The thermal pulp vitality tests to heat and cold and electric tests are not completely reliable for detection of pulp necrosis ${ }^{25}$. Discoloration of the tooth crown is also not 
pathognomonic of pulp necrosis, because pulp hemorrhage after trauma might lead to the overflow of erythrocytes and byproducts of their decomposition to the surrounding tissue, making the tooth crown pinkish or grayish. After reestablishment of normal circulation at the region, these byproducts could be eliminated and the normal color of the tooth crown could be recovered ${ }^{4}$. The presence of radiolucent areas at the periapical region of luxated teeth may represent intermediate stages of pulp repair, not necessarily indicating pulp necrosis ${ }^{2}$. A histobacteriological assessment of removed pulps with clinical diagnosis of pulp necrosis after dental trauma revealed that $9 \%$ to $14 \%$ of these pulps presented potential for repair, and thus they should not have been removed ${ }^{3}$.

The presence of microorganisms in the root canal system should also be evaluated, which may definitely impair the processes of revascularization and regeneration of the ischemic pulp tissue after luxation ${ }^{14}$. Previous studies demonstrated that, in traumatized teeth with intact pulp chambers, the necrotic pulp may be either infected or free of microorganisms for a long time, which raises questions on the actual need of endodontic intervention ${ }^{9,11,21,27}$.

The purpose of the present study was to evaluate the presence of microorganisms and analyze microscopically the pulp of traumatized human permanent teeth with intact crowns and clinical diagnosis of pulp necrosis.

\section{MATERIAL AND METHODS}

\section{Clinical Procedures}

The study sample was composed of 20 patients from the research and extension project "Teeth should be in the mouth", conducted by the Dental School of the Federal University of Goiás (Brazil) and approved by the respective Institutional Review Board.

The inclusion criteria comprised teeth with history of trauma to the periodontal tissues (subluxation -8 teeth, extrusive luxation -3 teeth, lateral luxation -2 teeth, and avulsion -7 teeth), presenting intact crowns and clinical diagnosis of pulp necrosis.

Teeth presenting root fracture, periapical lesion, periodontal pocket, resorptions or root canal obliteration were excluded.

The following clinical data were recorded: a) type of trauma; b) date of trauma and time elapsed between dental trauma and the onset of endodontic intervention; c) crown shade; d) response to thermal and electric pulp vitality tests (PVT) (positive or negative) - (heat: a piece of heated guttapercha was applied to the center of the buccal aspect of the affected tooth, previously lubricated with petroleum jelly, with cotton roll isolation; cold: a cold spray at $-50^{\circ} \mathrm{C}$ (Endofrost;Roeko, Langenau, Germany) was applied to the center of the buccal aspect of the affected tooth, with cotton roll isolation; electric test: this was performed with the Endoanalyser device (Kerr Corporation, Orange, CA, USA), placing the electrode tip on the center of the buccal aspect lubricated with fluoride gel, with cotton roll isolation, and recording the response value); e) response to vertical (VPT) and horizontal (HPT) percussion tests (positive or negative); f) pain upon palpation (absent or present); g) symptomatology (absent or present); h) mobility (normal or increased).

All these clinical data were also recorded for the contralateral teeth, in order to check the response pattern of each patient.

Radiographic examination was performed with a film holder for adult patients (Indusbelo, Londrina, Brazil) and Insight radiographic film (Eastman Kodak Company, Rochester NY, USA), with the same X-ray machine, to assure constant power and current intensity. The radiographs were analyzed in an indirect light environment, at a different moment than clinical examination. Black cardboard masks were fabricated to improve the observation of radiographs on the film viewer.

After clinical diagnosis of pulp necrosis based on the association of at least three clinical criteria, the teeth were submitted to endodontic treatment. For each selected tooth, rubber dam was placed and antisepsis was performed with $1 \%$ sodium hypochlorite solution (Probem, Catanduva, SP, Brazil). Coronal opening was limited to trepanation of the pulp chamber. Following, microbiological collection of the root canal was performed, based on previous studies ${ }^{17,26}$, with the aid of autoclaved absorbent paper points n. 20 (Tanari, Manacapuru, AM, Brazil). Three paper points were employed for each tooth. Each point was introduced in the root canal up to the provisional working length with sterile pliers and kept for $30 \mathrm{~s}$, removed and placed in a test tube containing Sørensen phosphate buffer solution (PBS). The samples were sent to the Department of Microbiology, Immunology, Parasitology and Pathology of the Institute of Tropical Pathology and Community Health of the Federal University of Goiás for microbiological processing.

When present, the pulp tissue was removed at the provisional working length with Hedström files (Maillefer, Ballaigues, Switzerland) of compatible size with the root canal, which was placed on an autoclaved cardboard and then in a flask containing $10 \%$ buffered formalin labeled with the patient number for later laboratory processing, which was performed at the Oral Pathology Laboratory of the Dental School of the Federal University of Goiás.

Each tooth was then prepared according to the protocol of endodontic treatment for traumatized teeth of the research and extension project "Teeth should be in the mouth", conducted by the Dental School of the Federal University of Goiás. The patients were followed at every 3 months by clinical and radiographic examination, until root canal obturation was indicated.

\section{Microbiological and Microscopic Processing}

For establishment of the number of colony forming units (cfu) of bacteria, the collected material was plated on chocolate agar (total bacterial counting), mitis salivarius agar (oral streptococci counting) and SB20 agar (mutans streptococci counting). The plated Petri dishes were incubated in anaerobiosis jars (microaerophilia) at $37^{\circ} \mathrm{C}$ for 
up to $96 \mathrm{~s}$. After the incubation period, the developed colonies were counted according to their macroscopic characteristics, with the aid of a stereomicroscope.

The removed pulp samples were processed in an automated histoprocessor (OMA-DM20), in which they were dehydrated with a series of increasing ethanol concentrations, cleared with xylol and immersed in liquid paraffin to obtain blocks. Serial 5.0-mm-thick sections were cut with a rotary microtome (model RM2155; Leica Instruments, Germany), placed on microscopic glass slides and routinely stained with hematoxylin and eosin (H.E).

The tissue sections were analyzed on a trinocular microscope (Axiostar Plus, Carl Zeiss, Jena, Germany), which allowed the investigation of possible morphological changes and related inflammatory processes in the pulp of traumatized teeth.

\section{Analysis of Results}

Linear Regression was used to evaluate the time elapsed from trauma until onset of endodontic intervention, as to the number of microorganisms in the root canal of traumatized teeth, at a significance level of $5 \%$. The diagnostic analysis was adopted to evaluate the sensitivity, specificity and accuracy of the vertical percussion test as to the presence of microorganisms in the root canals of these teeth. The sections stained with H.E. were analyzed subjectively, based on histological study of pulps extirpated after luxation injuries ${ }^{3}$, to evaluate the occurrence of collagen hyalinization, presence of blood vessels, cell nuclei and nerve bundles.

\section{RESULTS}

\section{Microbiological Evaluation}

Among the 20 root canal samples obtained from traumatized teeth, only $3(15 \%)$ did not present microbial development. The following microorganisms were isolated: oral streptococci $(31 \%)$, facultative anaerobic bacteria -

TABLE 1- Distribution of the number of traumatized teeth $(n=20)$ according to the time elapsed between dental trauma and the onset of endodontic intervention

\begin{tabular}{lc} 
Period & Number of teeth (\%) \\
\hline 15 days -3 months & $14(70 \%)$ \\
4 months -6 months & $2(10 \%)$ \\
$>7$ months & $4(20 \%)$ \\
\hline
\end{tabular}

TABLE 2- Analysis of vertical percussion test (VPT) in relation to the presence of microorganisms in the root canal

\begin{tabular}{lrrrr}
\hline $\begin{array}{l}\text { Presence of } \\
\text { microorganisms }\end{array}$ & \multicolumn{2}{c}{ VPT (+) } & \multicolumn{2}{l}{ VPT (-) } \\
& $\mathbf{n}$ & $\%$ & $\mathbf{n}$ & $\%$ \\
\hline & 3 & 20 & 0 & 0 \\
No & 12 & 80 & 5 & 100 \\
Yes & 15 & 100 & 5 & 100 \\
Total & &
\end{tabular}

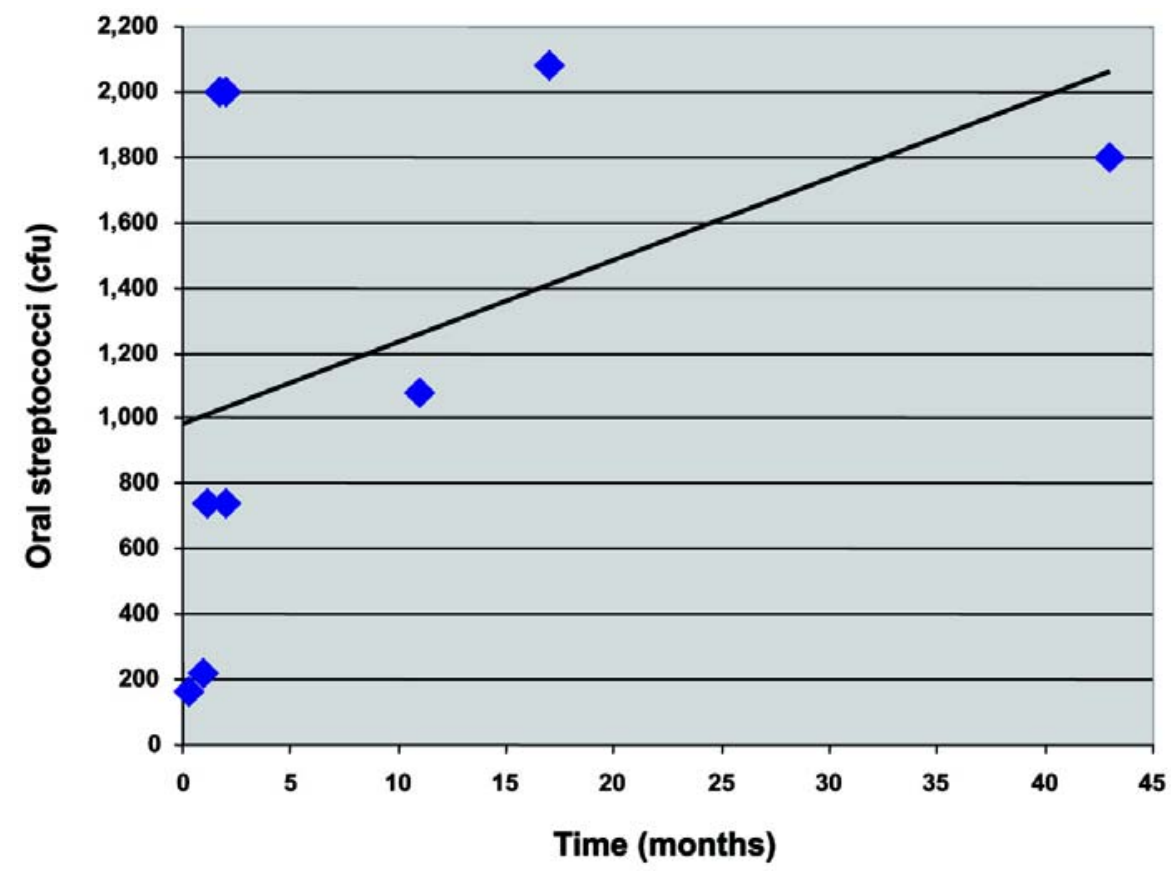

FIGURE 1- Graph demonstrating the correlation between the number of colony forming units (cfu) of oral streptococci and the time elapsed from dental trauma until endodontic treatment 
Gram-positive cocci, alpha-hemolytic Gram-positive cocci and alpha-hemolytic Gram-positive bacilli (52\%), Staphylococcus sp (6.7\%) and sporulated Gram-positive bacilli (10.3\%). More than one type of microorganism was observed in 12 teeth.

The time elapsed from the occurrence of dental trauma up to onset of endodontic intervention ranged from 15 days to 31 months (Table 1). This period was not statistically significant $(p=0.591)$ in relation to the number of microorganisms present in the root canal, when all types

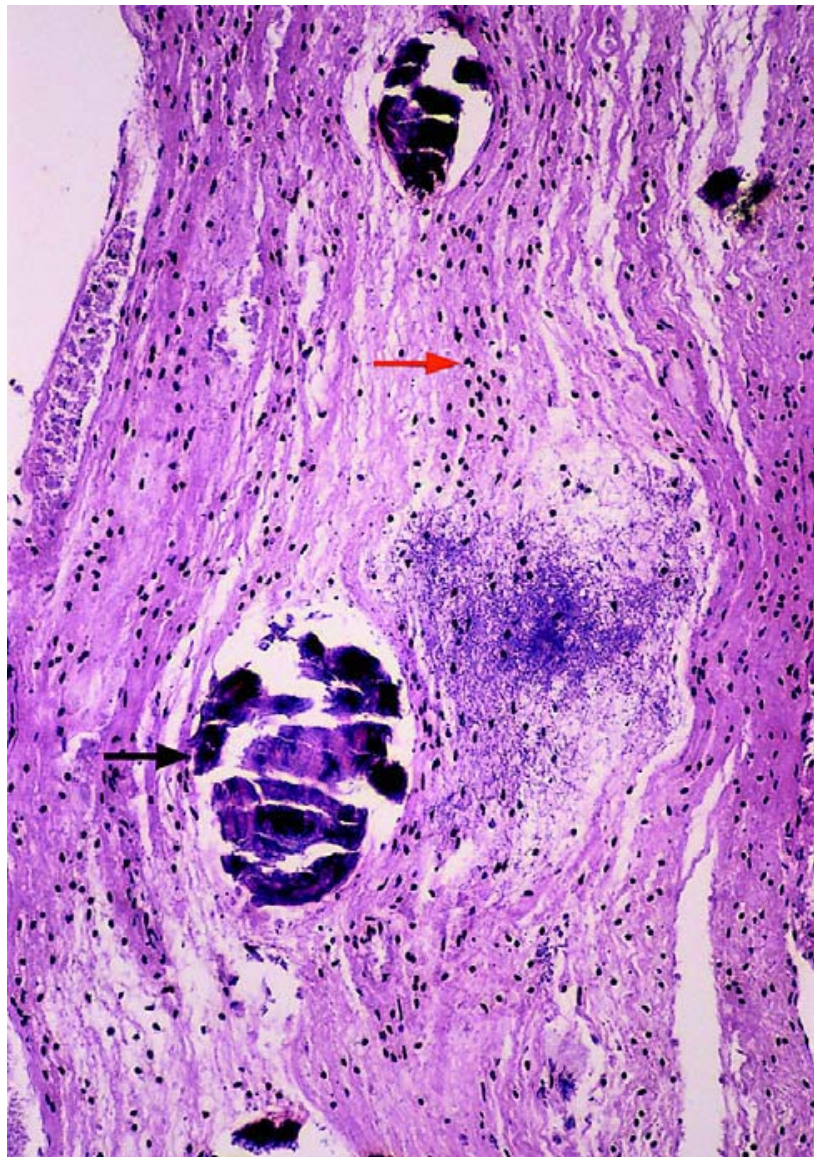

FIGURE 2- Pulp with partial necrosis, areas with preserved cell nuclei (red arrow) and calcification (black arrow) (H.E., original magnification 200X) were analyzed in combination. However, the individual analysis of each type of microorganism revealed that the time elapsed from dental trauma up to endodontic intervention was statistically significant $(\mathrm{p}=0.047)$ as to the number of oral streptococci isolated from the root canal, with an increase in this number with the increase in the time period (Figure 1).

The vertical percussion test was analyzed as to the presence of microorganisms in traumatized teeth, exhibiting high sensitivity (80\%) (Table 2 ).

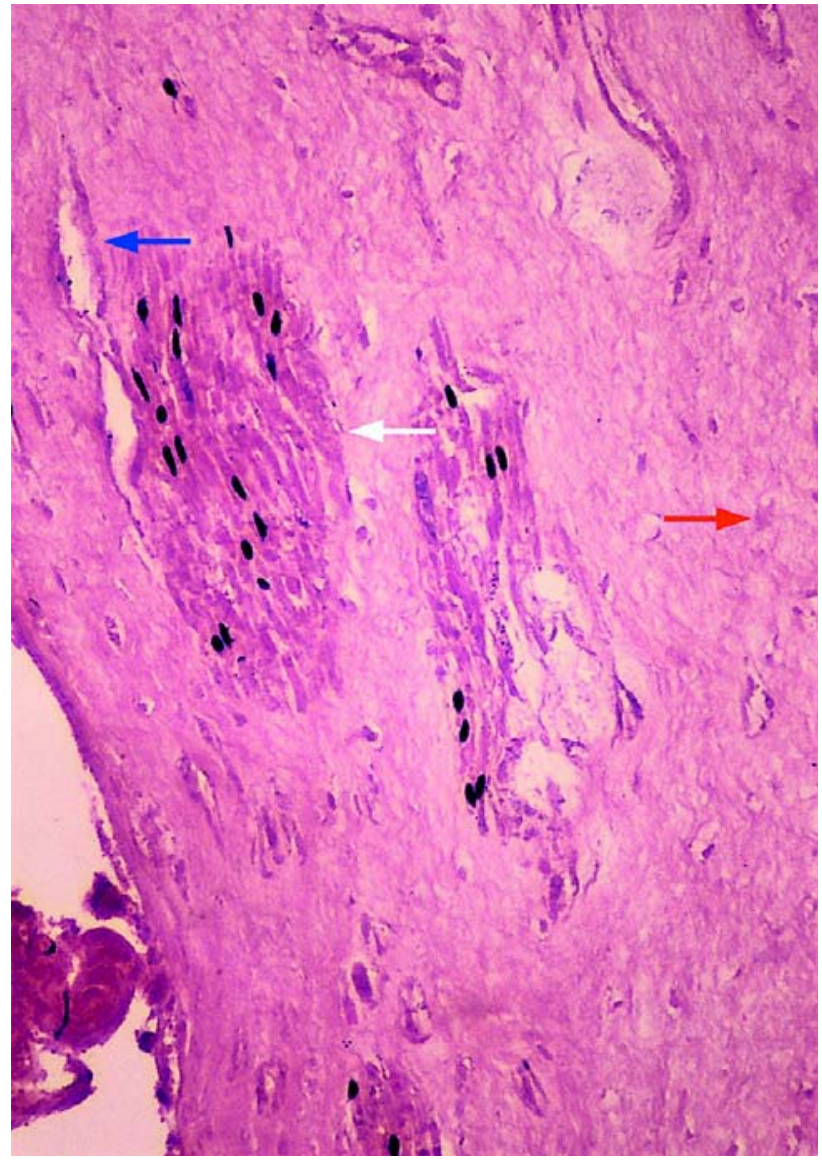

FIGURE 3- Pulp with complete necrosis, degeneration of blood vessels (blue arrow), cell nuclei (red arrow) and nerve bundles (white arrow) (H.E., original magnification 400X)

TABLE 3- Analysis of clinical criteria employed for the diagnosis of pulp necrosis

\begin{tabular}{|c|c|c|c|c|c|c|c|c|}
\hline \multicolumn{9}{|c|}{ Clinical criteria } \\
\hline & $\begin{array}{l}\text { Crown } \\
\text { shade }\end{array}$ & $\begin{array}{l}\text { PVT } \\
\text { cold (-) }\end{array}$ & $\begin{array}{l}\text { PVT } \\
\text { heat (-) }\end{array}$ & $\begin{array}{l}\text { PVT } \\
\text { electric (-) }\end{array}$ & VPT (+) & HPT (+) & $\begin{array}{l}\text { Pain upon } \\
\text { palpation }\end{array}$ & $\begin{array}{l}\text { Increased } \\
\text { mobility }\end{array}$ \\
\hline $\begin{array}{l}\text { Teeth with } \\
\text { diagnosis of } \\
\text { pulp necrosis } \\
(\%)\end{array}$ & 30 & 85 & 90 & 50 & 75 & 55 & 20 & 50 \\
\hline
\end{tabular}

(-): negative response; (+): positive response; PVT: pulp vitality test; VPT: vertical percussion test; HPT: horizontal percussion test. 


\section{Microscopic Analysis}

Among the 20 traumatized teeth selected for this study, 3 did not present pulp tissue, being characterized as complete autolysis. Pulp samples from the remaining 17 teeth were analyzed, among which $3(15 \%)$ presented partial necrosis (Figure 2) and 14 (70\%) exhibited complete necrosis (Figure $3)$. In the pulps with partial necrosis, despite the evident necrotic and degenerative phenomena in some regions, nerve bundles could be observed in some areas, as well as collagen, cell nuclei and blood vessels, but without chance of regeneration. The pulps with complete necrosis demonstrated partial or complete collagen hyalinization, and absence or advanced degeneration of blood vessels, cell nuclei and nerve bundles.

Based on the microscopic findings, none of the clinical criteria employed in the present study was pathognomonic. However, the pulp sensitivity tests to heat $(90 \%)$ and cold $(85 \%)$ and the vertical percussion test $(75 \%)$ were more reliable than the others (Table 3 ).

\section{DISCUSSION}

The present study was conducted on patients with permanent teeth with history of trauma to the periodontal tissues (subluxation, extrusive luxation, lateral luxation and avulsion), with intact crowns and clinical diagnosis of pulp necrosis. The groups did not have similar numbers of samples because the study did not aim to correlate the type of dental trauma with the occurrence of pulp necrosis, but rather to verify if the clinical and radiographic criteria available for this diagnosis in traumatized teeth are concordant with their microbiological and microscopic characteristics.

The teeth did not present carious lesions, restorations, fractures or periodontal disorders, which might represent possible sources of microbial invasion in the pulp tissue ${ }^{1,24}$. Teeth with periapical lesion or resorptions were also excluded because the presence of free microorganisms in the root canal, adhered to the root canal walls and/or penetrating the dentinal tubules has been demonstrated in these cases $^{22,23}$.

Collection of material from traumatized teeth to determine the number of colony forming units of bacteria was performed by sequential introduction of three sterile absorbent paper points in the root canal for $30 \mathrm{~s}$. The procedure was based on studies conducted by Engstrom ${ }^{17}$ and Serene and $\mathrm{McDonald}^{26}$, in which the most reliable indication for collection with positive culture was obtained by utilization of three paper points for this time period.

The development of pulp necrosis in traumatized teeth is determined by three factors: presence of microorganisms, which may impair the processes of revascularization and pulp regeneration ${ }^{14}$; type of trauma, which is associated with the damage to the pulp-periodontal complex; and the stage of root development, which is related to the repair capacity ${ }^{5,6}$. The concussion and subluxation, which cause minimal aggression to the periodontium and the pulp, present limited risk of developing pulp necrosis. Conversely, this risk is increased in avulsions and extrusive, lateral and intrusive luxation due to the greater damage to the pulp, periodontal ligament and alveolar bone ${ }^{5,6}$. Teeth with incompletely formed roots also presented reduced risk to develop pulp necrosis due to the greater possibility of pulp revascularization ${ }^{5,6,14}$.

Among the 20 samples obtained from the root canals of traumatized teeth, 17 (85\%) exhibited microbial development. This result is higher than those reported by Arwill, et al. ${ }^{9}$ and Bergenholtz ${ }^{11}$, who observed microbial growth in traumatized teeth in $28.6 \%$ and $64 \%$ of cases, respectively, and lower than those of Taklan ${ }^{27}$ and Le Goff, et al. ${ }^{21}$, who found microbial development in traumatized teeth with intact pulp chambers in $100 \%$ and $90 \%$ of cases, respectively.

The time elapsed from dental trauma up to endodontic intervention in the selected teeth was also analyzed, because little is known on when pulp necrosis may be accurately diagnosed in these teeth. The time period between dental trauma and onset of endodontic intervention in the present study ranged from 15 days to 31 months. The teeth indicated for treatment after 15 days involved the avulsion of teeth with completely formed roots, since Andreasen ${ }^{4}$ stated that pulp necrosis is the only outcome expected in these cases. Moreover, in 14 teeth (70\%), the clinical diagnosis of pulp necrosis was performed in up to three months. This result agrees with the report of Andreasen ${ }^{4}$ and is close to the findings of Jacobsen ${ }^{19}$, namely four months. Conversely, Barkin ${ }^{10}$ stated that the vitality of traumatized teeth cannot be predicted in the first three months. According to Andreasen and Vestergaard-Pedersen ${ }^{6}$ and Andreasen ${ }^{4}$, different chronological patterns are observed for different types of trauma; pulp necrosis may occur after three months or even after two years in cases of lateral and intrusive luxation.

Analysis of this time length also aimed to investigate the correlation between it and the number of microorganisms present in the root canal. Even though Bergenholtz ${ }^{11}$ reported that the number of microorganisms present in the root canal was increased with the increase in the time elapsed from dental trauma up to endodontic intervention, in the present study this increase was not statistically significant as to the total number of isolated microorganisms, except for oral streptococci. This fact may be explained, since rupture of the nerve and blood supply to the pulp after dental trauma may lead to ischemia and thus jeopardize the pulp to microbial invasion. Since oral streptococci are predominant and are always present in the oral cavity, root canal colonization by them is facilitated. Also, these microorganisms are able to survive in environments with few nutrients ${ }^{20}$.

Some hypotheses have been proposed to explain how the microorganisms may reach the intact pulp chamber of traumatized teeth. This origin would be related to the rupture of blood vessels in the periodontium, allowing the penetration of microorganisms through the gingival sulcus, by the collateral or even the systemic blood circulation ${ }^{18,27}$, 
and through the dentinal tubules in cases of coronal fracture associated or not with luxation ${ }^{11}$. This invasion might also occur due to penetration of microorganisms through cracks present in the enamel and dentin of avulsed teeth ${ }^{22}$. For these teeth, microorganisms coming from the oral cavity or the root surface, contaminated during the extra-alveolar time, might reach the apical region of the replanted tooth through the blood clot present in the alveolus before replantation or formed during the initial healing periods, since the blood clot is an excellent substrate for microbial proliferation ${ }^{15}$.

The present results revealed that $85 \%$ of pulps submitted to light microscopy analysis presented necrosis, being $15 \%$ with partial necrosis without possibility of repair and $70 \%$ with complete necrosis. The remaining 3 cases $(15 \%)$ exhibited complete autolysis, adding up to $100 \%$ of nonvital teeth. These results disagree with the findings of Arwill, et al. ${ }^{9}$, who investigated a sample of 25 pulps removed from teeth with clinical diagnosis of pulp necrosis after trauma and observed that only $56 \%$ were really necrotic on microscopic examination. Cipriano and Walton ${ }^{12}$, in turn, did not confirm microscopically the clinical diagnosis of pulp necrosis after trauma in $88 \%$ of analyzed teeth. Also, Andreasen ${ }^{2}$ conducted microscopic evaluation of a sample of 66 teeth with clinical diagnosis of pulp necrosis and observed that 9 to $14 \%$ of removed pulps still presented possibility of repair and should not have been removed.

This difference between the present results and the reports in the literature ${ }^{2,9,17}$ could be first assigned to the year of accomplishment of studies, considering the limitation of diagnostic methods available at the time for establishment of pulp necrosis after trauma, as well as the utilization of isolated clinical criteria adopted to diagnose pulp necrosis in the most recent studies.

In the present study, the criteria adopted for clinical diagnosis of pulp necrosis comprised discoloration of the crown, negative response to thermal and electric pulp vitality tests, positive response to vertical and horizontal percussion tests, pain upon palpation and increased mobility ${ }^{5,6,19}$. The clinical diagnosis of pulp necrosis of traumatized teeth was only achieved if at least three of these clinical criteria were present. None of the criteria was pathognomonic (100\%) to diagnose pulp necrosis in traumatized teeth. However, the heat (90\%), cold (85\%) and vertical percussion (75\%) pulp vitality tests were more reliable than the others, also when compared to the electric test (50\%). Peterson, et al. ${ }^{25}$ also observed the superior outcomes of thermal pulp vitality tests $(87.5 \%)$ compared to the electric test $(62.5 \%)$ in the diagnosis of pulp necrosis. The last test has also been associated with false-negative responses in traumatized teeth, because it stimulates the nerve fibers in the pulp to produce response, yet it does not provide information on the blood supply of the pulp. Since the blood supply presents faster reestablishment than the nerve supply of the pulp after dental trauma, the tooth may be vital yet insensitive ${ }^{13,19}$. However, this is still an important and non-subjective clinical criterion, because it provides a value that may be monitored. Discoloration of the crown after dental trauma should not be expected as a definite sign of pulp necrosis, yet it should be associated with the other clinical criteria for diagnosis ${ }^{5,13}$.

The vertical percussion test has been a clinical criterion with simple accomplishment and good results. Previous studies revealed that teeth with infected necrotic pulps exhibited higher sensitivity to percussion when compared to teeth with non-infected necrotic pulps, indicating that a positive response to the vertical percussion test would be associated with pulp necrosis with presence of microorganisms $\mathrm{s}^{3,5}$. This finding was confirmed in the present study, in which this test exhibited high sensitivity (80\%) for detection of microorganisms in the root canals of traumatized teeth.

In summary, even though the inclusion and exclusion criteria determined a limited sample (20 cases), the present study demonstrated that complete and careful follow up of the traumatized tooth, by the association of at least three clinical tests well performed and interpreted, may determine a more accurate diagnosis of pulp necrosis, as demonstrated by the microscopic and microbiological findings. Notwithstanding, the present results agree with those of other authors who state that full understanding of pulp necrosis occurring after trauma requires further microscopic and microbiological studies on larger samples and using standardized methodologies.

\section{CONCLUSIONS}

Under the tested conditions and within the limitations of the employed methodology, the following conclusions may be drawn: 1. Regarding the microbiological results, $85 \%$ of teeth presented microorganisms in the root canal, despite the presence of intact crowns; 2. Regarding the microscopic findings, $100 \%$ of traumatized teeth exhibited pulp necrosis; 3 . The pulp vitality tests to heat, cold and vertical percussion were the most reliable to diagnose pulp necrosis in traumatized teeth.

\section{REFERENCES}

1- Abou-Rass M, Bogen G. Microorganisms in closed periapical lesions. Int Endod J. 1998;31:39-47.

2- Andreasen FM. Transient apical breakdown and its relation to color and sensibility changes after luxation injuries to teeth. Endod Dent Traumatol. 1986;2:9-19.

3- Andreasen FM. Histological and bacteriological study of pulps extirpated after luxation injuries. Endod Dent Traumatol. 1988;4:17081.

4- Andreasen FM. Pulpal healing after luxation injuries and root fractures in the permanent dentition. Endod Dent Traumatol. 1989;5:111-31.

5- Andreasen FM. Transient root resorption after dental trauma: the clinician's dilemma. J Esthet Restor Dent. 2003;15:80-92.

6-Andreasen FM, Vestergaard-Pedersen B. Prognosis of luxated permanent teeth: the development of pulp necrosis. Endod Dent Traumatol. 1985;1:207-20. 
7- Andreasen JO. Challenges in clinical dental traumatology. Endod Dent Traumatol. 1985;1:45-55

8- Andreasen JO, Andreasen FM, Andersson L. Textbook and color atlas of traumatic injuries to teeth. Oxford: Blackweel Munksguard; 2007.

9- Arwill T, Henschen B, Sundwall-Hagland I. The pulpal reaction in traumatized permanent incisors in children aged 9-18. Odontol Tidskrift. $1967 ; 1: 130-47$

10- Barkin PR. Time as a factor in predicting the vitality of traumatized teeth. ASDC J Dent Child. 1973;40:188-92.

11- Bergenholtz G. Microorganisms from necrotic pulp of traumatized teeth. Odontol Revy. 1974;25:347-58.

12- Cipriano TJ, Walton RE. The ischemic infarct pulp of traumatized teeth: a light and electron microscopy study. Endod Dent Traumatol. 1986;2:196-204.

13- Cohenca N, Karni S, Rotstein I. Transient apical breakdown following tooth luxation. Dent Traumatol. 2003;19:289-91.

14- Côrtes MIS, Sheiham A, Marcenes W. Impact of traumatic injuries to the permanent teeth on oral-health related quality of life of 12 to 14 year old Brazilian schoolchildren. Community Dent Oral Epidemiol. 2002;30:193-8

15- Cvek M, Cleaton-Jones P, Austin J, Lownie J, Kling M, Fatti P. Pulp revascularization in reimplanted immature monkey incisors-predictability and effect of antibiotic systemic prophylaxis. Endod Dent Traumatol. 1990;6:157-69.

16- Dumsha T, Hovland EJ. Pulpal prognosis following extrusive luxation injuries in permanent teeth with closed apexis. J Endod. 1982;8:410-2.

17- Engstrom B. The significance of the number of absorvent paper points to positive endodontic cultures. Odontol Revy. 1966;17:216-21.

18- Grossman LI. Origin of microorganisms in traumatized, pulpless, sound teeth. J Dent Res. 1967;46:551-3.

19- Jacobsen I. Criteria for diagnosis of pulp necrosis in traumatized permanent incisors. Scand J Dent Res. 1980;88:306-12.

20- Koneman EW. Color atlas and text book of diagnostic microbiology. Philadelphia: Lippincott Willians; 1997.

21- Le Goff A, Bunetel L, Mouton C, Bonnaure-Mallet M. Evaluation of root canal bacteria and their antimicrobial susceptibility in teeth with necrotic pulp. Oral Microbiol Immunol. 1997;12:318-22.

22- Love RM. Bacterial penetration of the root canal of intact incisor teeth after a simulated traumatic injury. Endod Dent Traumatol. $1996 ; 12: 289-93$

23- Nair PNR. Light and electron microscopic studies of root canal flora and periapical lesions. J Endod. 1987;13:29-39.

24- Paul BF, Hutter JW. The endodontic-periodontal continum revisited: new insights into etiology, diagnosis and treatment. J Am Dent Assoc. 1997;128:1541-8.

25- Petersson K, Soderstrom C, Kiani-Anaraki M, Lévy G. Evaluation of the ability of thermal and electrical tests to register pulp vitality. Endod Dent Traumatol. 1999;15:127-31.

26- Serene TP, McDonald ED. Endodontic culturing: a statistical study. J Am Dent Assoc. 1969;78:1013-5.

27- Taklan S. A bacteriological study of pulp of intact non-vital teeth. J Br Endod Soc. 1974;1:75-7. 